Gutiérrez-Villar, B., Alcaide-Pulido, P. y Carbonero-Ruz, M. (2017). ¿Cómo ven la universidad privada los estudiantes preuniversitarios? Un estudio exploratorio mediante la combinación de Redes Semánticas Naturales y despliegue multidimensional. Revista de Investigación Educativa, 35(2), 519-535

DOI: http://dx.doi.org/10.6018/rie.35.2.257801

\title{
¿Cómo ven la universidad privada los estudiantes preuniversitarios? Un estudio exploratorio mediante la combinación de Redes Semánticas Naturales y despliegue multidimensional
}

\author{
How Do Pre-University Students See Private Universities? An \\ Exploratory Study by Combination of Natural Semantic Networks \\ and Multidimensional Unfolding
}

\author{
Belén Gutiérrez-Villar, Purificación Alcaide-Pulido y Mariano Carbonero-Ruz \\ Universidad Loyola Andalucía
}

\begin{abstract}
Resumen
Se presenta una investigación en la que se estudian los atributos determinantes en la construcción de imagen de la universidad privada, de forma genérica sin alusiones a marcas, recurriendo a una muestra de conveniencia de preuniversitarios en Andalucía. Como metodología se propone una investigación exploratoria con la combinación de dos técnicas poco habituales: la técnica Redes Semánticas Naturales, y una técnica cuantitativa de escalamiento denominada despliegue multidimensional. Los resultados apuntan hacia la presencia de dos dimensiones diferentes: asociados a los resultados esperados de la universidad privada (formación, empleo e idioma) y según la implicación requerida del discente (esfuerzo e intercambios) y se perciben diferencias según el sexo y la naturaleza del centro de pertenencia del alumnado. Además, se aportan evidencias de las ventajas de la combinación de técnicas como mecanismo de triangulación, pues utilizando procedimientos de análisis diferentes sobre el mismo fenómeno se constata una mejor comprensión del problema de investigación.
\end{abstract}

Palabras clave: Imagen de universidad; RSN; PRESFCAL.

Correspondencia: Belén Gutiérrez Villar, belengut@uloyola.es, Universidad Loyola Andalucía, Calle Escritor Castilla Aguayo 4, 14004 Córdoba postal. 


\begin{abstract}
This research studies the determinant attributes of the construction of the perception of private universities generically without referencing brands using a convenience sample of high school seniors in Andalusia. The methodology proposed is an exploratory research study combining two non-habitual techniques: natural semantic networks, a quantitative technique, and multidimensional unfolding, a scaling quantitative technique. The results point to the presence of two different dimensions: the associations with the expected results from private university (training, employment and language) and the required involvement of the student (effort and exchanges) and differences perceived according to sex and the nature of the student's center of belonging. In addition, there is evidence of the advantages of combining techniques as a triangulation mechanism. Better comprehension of a research problem is verified by using different analysis procedures about the same phenomenon.
\end{abstract}

Keywords: perception of university; NSN; PREFSCAL.

\title{
Introducción
}

La integración global de la Educación Superior ha ampliado el ámbito competitivo en la mayor parte de países desarrollados y se compite por los estudiantes y los recursos económicos más allá del entorno geográfico cercano (Luque-Martínez y Del Barrio-García, 2007; Harrison-Walker, 2011). Unido a ello, en España la rivalidad competitiva se ha acentuado debido también a varios fenómenos de índole interno que provocan un desajuste entre la oferta de plazas y sus demandantes. Mientras los preuniversitarios disminuyen (fruto del descenso de la natalidad y de una tasa de abandono escolar temprano que dobla la media de la UE) en los últimos años la oferta de universidades ha aumentado en número y se han ampliado los catálogos de títulos que estas ofertan. Sin ser exhaustivos en este tema, baste indicar que, según las cifras del Instituto Nacional de Estadística, en el curso 2000-2001 había en España un total de 66 universidades (48 públicas y 18 privadas), mientras que los datos del Ministerio de Educación, Cultura y Deporte (curso 2014-2015) se menciona un total en 83 universidades (50 públicas y 33 privadas).

En este entorno, conocer cómo se genera la imagen de la universidad en los diferentes grupos de interés o stakeholders es un tema que suscita un amplio interés pues, ante este incremento de la competencia, en la que se mezclan agentes públicos y privados, es conveniente una mejor comprensión de los deseos y necesidades de los demandantes de los servicios universitarios. Que estos deseos sean los que orienten a las universidades en el diseño de sus estrategias y les posibilite abrirse un hueco en el mercado, con ofertas y marcas universitarias diferenciadas.

Se exploran en este trabajo las ventajas de una tendencia metodológica en auge, como es la integración de métodos de investigación en un mismo estudio, enfoque conocido como métodos mixtos de investigación. Esta forma de trabajar permite al investigador evitar optar entre dos aproximaciones, para centrarse en determinar cómo se pueden combinar las fortalezas de cada enfoque a través de una aproximación combinada (Azorín, Gamero, Moliner, Ortega y Guilló, 2012). Así, primero a través de las Redes Semánticas Naturales se aproxima el significado psicológico que para los 
jóvenes preuniversitarios tienen las universidades privadas, identificando los atributos principales sobre los que opera la percepción de los individuos y posibles diferencias atribuibles a sus características personales (sexo y naturaleza del centro en el que estudian el bachillerato) para, a continuación, desarrollar y validar los resultados con una técnica netamente cuantitativa, en concreto el despliegue multidimensional.

\section{Contextualización de la imagen de universidad}

A diario experimentamos una gran cantidad de imágenes del mundo exterior de muy diversa índole, y, en consecuencia, nuestra mente está repleta de imágenes mentales, impresiones, ideas y demás representaciones asociadas; aunque a priori pueda resultar un término cotidiano, la palabra imagen es utilizada con varias acepciones. Cuando descendemos al ámbito de la gestión empresarial, las decisiones sobre la imagen afectan a dos acepciones genéricas: una acepción "material" alude a fenómenos exteriores perceptibles a nivel visual (por ejemplo, el logotipo, la estética del establecimiento, grafismos, etc.); la otra, en la que pivota este trabajo, sería "perceptual" y considera la imagen como una representación psíquica e ideológica de los significados y valores de un objeto en la mente de una persona o de un colectivo. Así, el concepto de imagen se define como el conjunto de creencias, actitudes, estereotipos, ideas, conductas relevantes o impresiones que una persona tiene y se puede referir no sólo a productos, sino también a personas u organizaciones no lucrativas (Kotler y Andreasen, 1996).

Cuando se profundiza en este concepto a un contexto concreto, está generalmente aceptada la posibilidad de distinguir entre diferentes niveles de análisis, desde un objeto amplio (por ejemplo, una nación, un sector industrial, una categoría de producto) hasta la imagen de una marca concreta. Pero no existe unanimidad, y dichos niveles no son coincidentes en número ni en el objeto analizado; se pueden encontrar autores defensores de cuatro niveles (Barich y Kotler, 1991) o cinco como Capriotti (1999). Aunque no exista coincidencia, sí se deriva de los diferentes enfoques la conveniencia de analizar la imagen de un producto, entendido como genérico, antes de descender a la interpretación de las imágenes que tendrían las diferentes marcas que se sitúan en una determinada categoría de producto, o sector de actividad.

Si se analizan las aportaciones empíricas, la revisión de 17 estudios publicados desde 2001 hasta la actualidad nos ha llevado a constatar una serie de aspectos interesantes, relativos al procedimiento metodológico y el tipo de stakeholders estudiados.

Por lo que respecta a la naturaleza de la metodología empleada, hemos distinguido entre trabajos monométodo (es decir, empleo de métodos solamente cuantitativos o cualitativos), frente a una metodología mixta (integración combinada de métodos cuantitativos y cualitativos). Predominan las aportaciones con metodologías monométodo cuantitativas; en menor medida aparecen trabajos mixtos, aunque estás metodologías tienen importantes ventajas frente a las monométodo pues, entre otras, permiten obtener una comprensión más completa del fenómeno estudiado, con una mayor confianza en los resultados y una mayor validez de las conclusiones (Azorín et al., 2012; 56). 
Tabla 1

Revisión de la naturaleza metodológica empleada en estudios recientes sobre imagen de universidad

\begin{tabular}{ll}
\hline Metodología & Autores \\
\hline Trabajos monométodo cuantitativos & Ivy (2001); Belanger, Mount y Wilson (2002); Beerli \\
& Palacio; Díaz Meneses y Pérez Pérez (2002); Sung \\
& y Yang (2008); Brown y Mazzarol (2009); Luque- \\
& Martínez y Del barrio-García (2009); Duarte, Alves \\
& y Raposo (2010); Pampaloni (2010); Joseph (2012); \\
& Hosseini y Nahad (2012); Cernicova, Dragomir y \\
& Palea (2015). \\
\hline Trabajos monométodo cualitativos & Iqbal, Rasli y Hassan (2012). \\
\hline Trabajos mixtos (combinan métodos cu- & Kazoleas, Kim y Moffit (2001); Arpan, Raney y \\
alitativos y cuantitativos) & Zivnuska (2003); Gray, Shyan Fam y Llanes (2003); \\
& Mackelo y Drūteikienè (2010). \\
\hline
\end{tabular}

Si se desciende a los grupos de interés o stakeholders, la complejidad a la que se enfrentan las universidades en este terreno es elevada, pues trabajos recientes llegan a identificar 21 grupos de interés (Mainardes, Alves y Raposo, 2013), lo que supone un enorme desafío en la gestión de las universidades. En los estudios revisados (tabla 1), se han encontrado un grupo amplio de estudios centrados únicamente en el grupo de los clientes o usuarios externos -los alumnos universitarios-, y en menor medida sobre los clientes internos -en nuestro caso el personal docente e investigador y personal de administración y servicios-. Solo los trabajos de Kazoleas et al. (2001) y Arpan et al. (2003), estudian también la imagen entre personas que no tienen una vinculación directa con la universidad. No se han localizado investigaciones sobre otros grupos de interés con los que se relacionan las universidades, como puede ser las opiniones de las empresas, de las familias con hijos en edades preuniversitarias, o de estos últimos, sobre los que se ha estudiado habitualmente el problema de la elección y nada prácticamente se ha dicho sobre cómo construyen su imagen sobre la universidad. Por último, añadir que predominan los trabajos en los que los encuestados tienen vinculación con la universidad pública, lo cual contribuye a que las instituciones con esta titularidad consigan la eficiencia y la rentabilización de los recursos depositados en ellas, pero faltan enfoques centrados en las universidades privadas, que se nos antoja aún más vital dado que su razón de ser y su supervivencia no se justifica si no aportan un valor diferencial al mercado (Ivy, 2010).

\section{Objetivos perseguidos}

Según lo anteriormente revisado, el problema de investigación es conocer de manera natural, sin apriorismos, cómo se construye la imagen genérica de universidad privada, en el colectivo de alumnos preuniversitarios. 
Este problema nos conduce a la formulación de objetivos que permitan extraer conclusiones cuantificables:

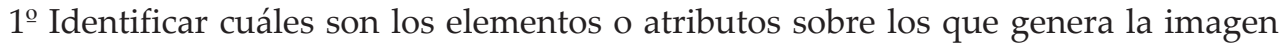
de universidad privada, de manera natural, el grupo de estudiantes preuniversitarios.

$2^{\underline{0}}$ Comprobar si pueden existir diferencias en la imagen perceptual, entre grupos de preuniversitarios, en razón a alguna característica individual, cuando se plantean la idea de universidad privada.

Pero, la revisión bibliográfica y la metodología propuesta también nos conducen a proponer tres cuestiones de investigación no cuantificables:

- Aportar más evidencias sobre las posibilidades de la técnica redes semánticas naturales (RSN) como método de captación de información en lugar de un cuestionario estructurado "a priori" por el investigador.

- Explorar la utilidad del escalamiento multidimensional, para determinar las dimensiones que utilizan los encuestados a la hora de evaluar un estímulo, sea cuál sea naturaleza de los datos utilizados y su interpretación perceptual.

- Aportar evidencias de las ventajas de la combinación de técnicas en un estudio exploratorio, obteniendo y analizando datos cuantitativos y cualitativos sobre el mismo fenómeno para una mejor comprensión del problema de investigación.

\section{Metodología, hipótesis planteadas y recogida de información}

\section{Metodología}

Esta investigación combina dos técnicas (RSN y despliegue multidimensional) para explorar los atributos alrededor de los cuales generan su imagen de universidad privada el colectivo de clientes potenciales (alumnos preuniversitarios). Siguiendo la clasificación propuesta por Azorín et al. (2012), se trataría de un diseño cuantitativocuantitativo, equivalente desde el punto de vista de la prioridad y simultáneos desde el punto de vista de recogida de la información.

Aunque en temas de medición de la imagen se recomiendan investigaciones mixtas como medio de obtención de un mejor resultado (Ortigueira-Sánchez y Ortigueira- Bouzada, 2000 y Gómez y Medina, 2013), hemos constatado tras la revisión bibliográfica que los procedimientos combinados en este trabajo, hasta donde conocemos, no han sido utilizados conjuntamente en ningún estudio previo.

Concretamente, el desarrollo de esta investigación comprende un primer paso donde, a través de las RSN, se recaba información subjetiva sin apriorismos, que permita indagar sobre los atributos principales presentes a nivel individual para generar la imagen cognitiva agregada (conjunto agregado de atributos que configuran la imagen del constructo universidad privada) a partir de los atributos que cada preuniversitario tiene.

Los promotores de esta teoría constatan que la información contenida en las palabras está organizada en forma de redes, en las cuales las palabras, eventos o representaciones forman relaciones que en conjunto producen significados (Figueroa, González y Solís, 1976). Dichos significados son dinámicos, lo que implica que las redes semánticas individuales pueden modificarse, permitiendo al individuo un ajuste diferencial al nuevo 
grupo de pertenencia o al mismo grupo con nuevos referentes sociales (Vera-Noriega, Eduardo Pimentel y Batista de Albuquerque, 2005). Así, se trata de un instrumento que puede resultar útil en las ciencias sociales pues permite estudiar cómo los sujetos forman sus representaciones sobre ideas, comportamientos, productos, etc. a través de sus propias redes.

Las principales ventajas de la técnica de redes semánticas naturales para su aplicación en esta investigación son dos. La primera es su consideración de "naturales", evitando la utilización de taxonomías artificiales creadas por los investigadores (Valdez, Gonzáles, Outhof y Gonzáles, 1998). Como segunda consideración, dicha técnica permite comparar dos o más grupos de acuerdo con el significado que le asigna cada grupo a ciertos conceptos claves, por lo que es una herramienta válida para conocer semejanzas y diferencias sobre los significados que tienen ciertos atributos, en un segmento de preuniversitarios determinado frente a otros.

En una segunda etapa se recurre a la validación de los resultados mediante la técnica multivariante del escalamiento multidimensional. No es la primera vez que observamos la aplicación de esta técnica en el ámbito de las universidades, ya que, por ejemplo, en el estudio de Sabiote, Pérez y Cano (2004) se analizan las disfunciones asociadas a la reforma de los nuevos planes de estudio en la universidad española. En líneas generales, estás técnicas permiten la representación de objetos considerados similares (disimilares) mediante puntos cercanos (distantes) en una representación espacial que descubre relaciones relevantes a los observadores y/o la estructura subyacente en los datos originales. De esta forma, si dos atributos de los identificados a través de las RSN son percibidos (o preferidos) de forma equivalente, los puntos a los que representan se aproximarán en el mapa perceptual, mientras que en el supuesto contrario su distancia en el espacio perceptual aumentará (Hair, Anderson, Tatham y Black, 1999, 548). En esta investigación se ha empleado, dentro de estas técnicas, el denominado despliegue multidimensional que intenta buscar una escala cuantitativa común que permita examinar visualmente las relaciones entre dos conjuntos de objetos. Entre los procedimientos de este tipo se encuentra el empleado en este trabajo, denominado PREFSCAL y cuya principal ventaja sobre otras alternativas existentes es su mayor eficacia para evitar las llamadas soluciones degeneradas ${ }^{1}$.

\section{Hipótesis planteadas}

De acuerdo con los objetivos principales de esta investigación expuestos ut supra, se plantean tres hipótesis principales, una de las cuales presenta dos sub-hipótesis, enunciadas a continuación.

- Hipótesis 1: Con independencia de las características sociodemográficas de los individuos objeto de estudio, la mayoría de los encuestados generan su imagen de universidad privada alrededor de un mismo grupo de atributos principales.

1 Una solución degenerada es una representación del conjunto investigado que es óptima desde el punto de vista numérico, pero de limitada validez real por su escasa interpretabilidad. 
- Hipótesis 2: No obstante, distintos subgrupos de encuestados generan su imagen alrededor de grupos de conceptos parcialmente diferenciados.

- Hipótesis 2a: El sexo femenino valora un mayor número de atributos que el masculino.

- Hipótesis 2b: El alumnado procedente de centros privados o concertados presenta diferencias en la valoración de los atributos principales frente a los que proceden de centros públicos.

- Hipótesis 3: Los atributos en virtud a los cuales se genera la imagen de universidad privada se pueden reagrupar en dos factores latentes, no mencionados directamente por los preuniversitarios.

\section{Recogida de la información}

\section{Población y muestra}

Como ha quedado indicado en párrafos anteriores, la población investigada la constituyen los estudiantes preuniversitarios de Andalucía, concretamente de segundo curso de bachillerato, si bien por cuestiones organizativas la recogida de información se limitó a Andalucía occidental (Cádiz, Huelva, Málaga, Córdoba y Sevilla), concretamente 877 alumnos que constituyen la muestra. El procedimiento de muestreo es no probabilístico pues los centros seleccionados no lo fueron al azar.

\section{Obtención de cuestionarios}

La recogida de información se llevó a cabo entre los meses de noviembre de 2012 y marzo de 2013.

Siguiendo las recomendaciones de Valdez et al. (1998), se elaboró para la recopilación de la información, un documento que incluía:

- Solicitud de datos individuales (sexo y centro actual donde cursa sus estudios), valores necesarios para la posible validación de las hipótesis.

- El documento disponía de diez líneas y dos columnas para el concepto estimulo "universidad privada".

Por lo que respecta al procedimiento, a los estudiantes se les repartió el formulario y verbalmente se les solicitó que escribieran 10 palabras definidoras para el constructo "universidad privada" y que las apuntasen según fueran llegando a su mente en la primera columna de la lista. Estas podían ser adjetivos, sustantivos, verbos y adverbios. A continuación, se les pidió que en la segunda columna las jerarquizaran en orden de importancia: a la palabra que considerase como la más importante para definir "universidad privada" se le asignaría el valor 1, a la siguiente el valor 2 y así, sucesivamente, hasta agotar todas las palabras escritas en la columna primera.

Mediante este procedimiento se recepcionaron un total de 877 formularios, de los cuales sólo se utilizaron 369 (ver figura 1); como valor de calidad para la aceptación se exigió un mínimo de siete palabras contestadas y que además presentasen la orde- 
nación por preferencia. Según las indicaciones de Zermeño, Arellano y Ramírez (2005) se procedió a unificar en un único término, los sinónimos, los plurales, las palabras iguales escritas con distinto género y derivadas de una misma matriz, sin alterar las puntuaciones originales dadas por los participantes.

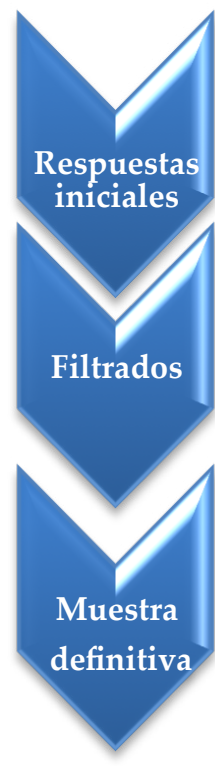

-Muestreo por conveniencia

-Alumnado de $2^{\circ}$ Bachillerato (centros públicos y privados) de Andalucía occidental

-Total respuestas iniciales: 877encuestas

-1ำ paso: eliminados 360 por contener menos palabras del valor establecido (menos de 7 términos)

- $2^{\circ}$ paso: suprimidos 148 cuestionarios mal cumplimentados

- Total de formularios válidos: 369

- Hombres 195

-Mujeres 174

- Público 184

-Privado 185

Figura 1. Ficha técnica del trabajo

\section{Resultados}

\section{Resultados de la RSN para el estímulo universidad privada}

Los cálculos más habituales utilizados en las Redes Semánticas Naturales se reflejan en la tabla 2. Aunque en varios de los estudios revisados no se especifica, el abordaje numérico del problema da origen a una pequeña modificación en los cálculos de la RSN que tienen que ver con el número de palabras definidoras; así, la diferencia sustancial entre la denominación de Redes Semánticas Naturales (Figueroa et al., 1976) frente a Redes Semánticas Naturales Modificadas (Reyes-Lagunes, 1993) es que la segunda da posibilidad de determinar el número de palabras definidoras y no establecerlo siempre en base a 10 términos. En este estudio los cálculos propuestos se ajustan a los procedimientos de las redes semánticas modificadas.

A continuación, se presentan los resultados más relevantes de la primera etapa de la investigación, exponiendo primero los resultados globales seguidos de los correspondientes a las submuestras atendiendo al criterio sexo y titularidad del centro en el que cursa el bachillerato. 
Comenzando con los aspectos relativos a la riqueza semántica, en total los preuniversitarios asociación a universidad privada un total de 206 palabras (tabla 3).

Tabla 2

Nomenclatura y procedimiento de cálculo de la Red Semántica Natural

\begin{tabular}{ll}
\hline Redes Semánticas Naturales & Redes Semánticas Naturales Modificadas \\
\hline Valor J = riqueza de la red. & $T R=$ tamaño de red. \\
$\begin{array}{l}\text { Total de palabras que generan los encuesta- } \\
\text { dos. }\end{array}$ & Total de palabras que generan los encuestados.
\end{tabular}

Valor M= peso semántico. PS= peso semántico.

Suma de la ponderación de la frecuencia de Suma de la ponderación de la frecuencia de las las definidoras por su jerarquización. definidoras por su jerarquización.

Conjunto SAM (Semantic Association Mem- NR= núcleo de red. ory) $=$ Cambia el procedimiento para obtenerlo. Para ello se seleccionan los elementos que definen al estímulo a través del punto de quiebre.

Formado por las 10 palabras definidoras con pesos semánticos más altos. $D S C=$ distancia semántica cuantitativa entre los diferentes palabras que integran el SAM.

Tabla 3

Tamaño y núcleo de red. Valor total y por grupos

\begin{tabular}{llll}
\hline Colectivo & Tamaño & TR & NR \\
\hline Hombres & 195 & 172 & 7 \\
Mujeres & 174 & 170 & 6 \\
Público & 184 & 163 & 6 \\
Privado & 185 & 165 & 8 \\
\hline Total & $\mathbf{3 6 9}$ & $\mathbf{2 0 6}$ & $\mathbf{7}$ \\
\hline
\end{tabular}

El segundo dato importante es la elección de los núcleos de las redes². Existen algunas diferencias atendiendo a la distribución en submuestras. El número de palabras

2 Como se explica previamente, las RSN modificadas determinan el núcleo de la red mediante el punto de quiebre, ligeramente modificado en nuestro caso. Para cada par de términos sucesivos se ha calculado la diferencia entre pesos semánticos. La variable así obtenida ha sido tipificada, y seleccionados los 
definidoras en cómputo global es 7; sin embargo, este núcleo de red alcanza un valor superior cuando la muestra se subdivide en razón al tipo de centro, llegando a 8 el número de palabras conformadoras de la red si el encuestado se encuentra cursando estudios en un bachillerato privado.

Se puede observar cómo el núcleo para el total de la muestra (ver tabla 4) está dominado por un concepto central que hace referencia al desembolso de la universidad privada, haciendo que la primera asociación sea con el término cara (NR), siendo este concepto el más puntuado siempre, sea cual sea la segmentación de la muestra. En segundo lugar, en cómputo global aparece el término formación, pero en este caso las mujeres le dan más peso que los hombres, para quienes ni siquiera es el segundo, que corresponde al esfuerzo. Además, la DSC es muy alta en el caso del colectivo femenino, lo cual indica que hay menos distancia con el término cara, están más próximos ambos términos en las puntuaciones femeninas (una distancia equivalente a 7 puntos en valor absoluto, mientras que el caso masculino se incrementa y se eleva hasta 37).

Tabla 4

Valores de la Red Semántica Natural para hombres, mujeres y totales

\begin{tabular}{lclllllll}
\hline \multicolumn{3}{c}{ Hombres } & \multicolumn{3}{c}{ Mujeres } & \multicolumn{3}{c}{ TOTAL } \\
\hline Palabra & PS & DSC & Palabra & PS & DSC & Palabra & PS & DSC \\
Cara & 768 & $100 \%$ & Cara & 614 & $100 \%$ & Cara & 1382 & $100 \%$ \\
Esfuerzo & 494 & $64 \%$ & Formación & 568 & $93 \%$ & Formación & 1049 & $76 \%$ \\
Formación & 481 & $63 \%$ & Esfuerzo & 341 & $56 \%$ & Esfuerzo & 835 & $60 \%$ \\
Intercambio & 388 & $51 \%$ & Prestigio & 338 & $55 \%$ & Intercambio & 707 & $51 \%$ \\
Empleo & 362 & $47 \%$ & Intercambio & 319 & $52 \%$ & Empleo & 679 & $49 \%$ \\
Idioma & 320 & $42 \%$ & Empleo & 317 & $52 \%$ & Prestigio & 652 & $47 \%$ \\
Prestigio & 314 & $41 \%$ & & & & Idioma & 593 & $43 \%$ \\
\hline
\end{tabular}

Al analizar los resultados agrupados por la otra característica investigada, pertenencia a un centro educativo privado o público (tabla 5), los resultados son más diferenciados. Así, aparece una mayor riqueza semántica en el grupo de alumnos de los bachilleratos privados, que es sensiblemente menor en el grupo de los estudiantes de la pública. Por lo que respecta a los términos mencionados, la palabra futuro sólo aparece con una valoración destacable en el primero de los grupos, que también asocian

términos hasta que sistemáticamente el valor tipificado se encuentre por debajo de 2 (lo que equivale a una pendiente poco pronunciada de la curva de pesos semánticos habitualmente empleada para la aplicación de este procedimiento). 
la universidad privada a la idea de diversión. Todo ello sin olvidar que la asociación entre universidad pública y empleo alcanza en este colectivo su mejor resultado y más llamativo aún si se compara con la opinión de los estudiantes de enseñanzas públicas, en cuyo caso dicha asociación no se ha contrastado entre los términos definidores del campo semántico. Por lo que respecta al conjunto de alumnado de bachillerato público, resalta la presencia del idioma asociado a la universidad privada y el mayor peso semántico de la palabra cara. Dicho término aun siendo el protagonista en todos los grupos estudiados, en este caso alcanza un valor claramente superior y su distancia semántica con la segunda palabra es más alta.

Tabla 5

Valores de la Red Semántica Natural para alumnado según naturaleza del centro de pertenencia (público o privado) y totales

\begin{tabular}{lcclcclcc}
\hline Centro Privado & PS & DSC & $\begin{array}{c}\text { Centro } \\
\text { público }\end{array}$ & PS & DSC & Total & PS & DSC \\
\hline Cara & 631 & $100 \%$ & Cara & 751 & $100 \%$ & Cara & 1382 & $100 \%$ \\
Formación & 519 & $82 \%$ & Formación & 530 & $71 \%$ & Formación & 1049 & $76 \%$ \\
Esfuerzo & 469 & $74 \%$ & Esfuerzo & 366 & $49 \%$ & Esfuerzo & 835 & $60 \%$ \\
Empleo & 403 & $64 \%$ & Intercambio & 366 & $49 \%$ & Intercambio & 707 & $51 \%$ \\
Intercambio & 341 & $54 \%$ & Idioma & 353 & $47 \%$ & Trabajo & 679 & $49 \%$ \\
Futuro & 319 & $51 \%$ & Prestigio & 352 & $47 \%$ & Prestigio & 652 & $47 \%$ \\
Prestigio & 300 & $48 \%$ & & & & Idioma & 593 & $43 \%$ \\
Diversión & 287 & $45 \%$ & & & & & & \\
\hline
\end{tabular}

\section{Resultados del análisis PREFSCAL de similitud-divergencia para el estímulo uni- versidad privada}

A continuación, se procede a un estudio de la similitud-divergencia entre los términos que componen el núcleo del campo semántico obtenido en apartados anteriores.

Mientras que el análisis de correspondencias o las técnicas de escalamiento o despliegue multidimensional, que son los medios a los que habitualmente se recurre para resolver este problema, se basan en las opiniones expresadas por un grupo de individuos ante un conjunto cerrado de objetos, las Redes Semánticas Naturales invitan a cada entrevistado a elegir libremente las palabras que posteriormente ordena. Valoración comparada es el elemento común en ambos casos, lista abierta o cerrada la diferencia. Para superar este inconveniente hemos elegido de la base de datos de entrevistados aquellos cuya lista de términos incluía al menos seis de los siete términos del núcleo 
semántico, que hemos vuelto a puntuar manteniendo la jerarquía del entrevistado, reasignando las puntuaciones de manera correlativa y asignando la peor a la palabra omitida cuando la había, pues la omisión supone un menor interés que el de cualquiera de las palabras mencionadas ${ }^{3}$.

Con este proceder hemos obtenido una lista de 28 conjuntos de puntuaciones cuyo contenido no desvirtúa la elección hecha por los entrevistados pero que permite la aplicación de las técnicas de que hablamos en párrafos anteriores con un tamaño muestral muy en línea con los tamaños muestrales habituales en su aplicación.

Hemos aplicado a esta muestra el procedimiento PREFSCAL (despliegue multidimensional), implementado en el software SPSS (versión 24), con objeto de obtener una representación bidimensional del espacio común final, lo que nos va a permitir visualizar las similitudes entre los significados de los términos del SAM para los sujetos considerados, así como la proximidad de cada uno de ellos al posicionamiento ideal de cada individuo. El diagrama bidimensional se presenta en la figura 2.

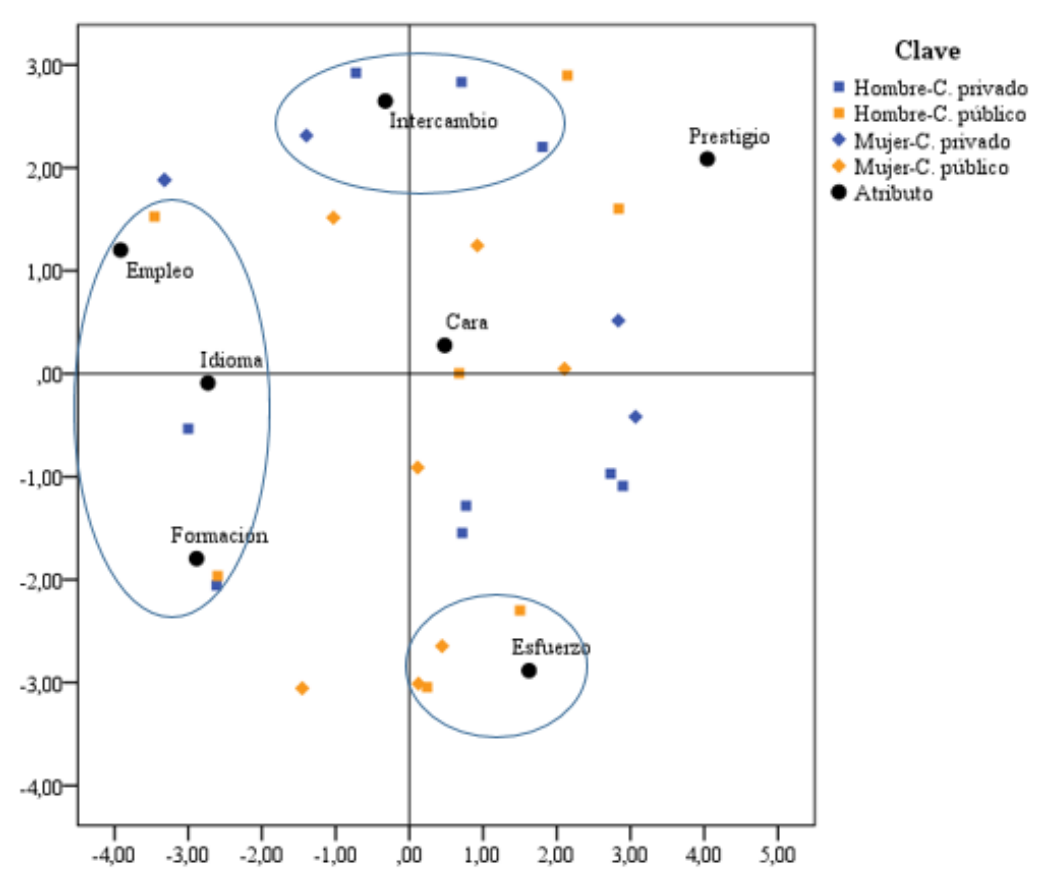

Figura 2. Diagrama bidimensional de términos resultantes del

Semantic Association Memory (SAM)

3 Supongamos un sujeto que, entre sus diez palabras elegidas ha mencionado seis del NR en las posiciones (ordenadas) 1, 3, 5, 7, 8 y 9, omitiendo la séptima. La ordenación asignada sería 1 a la que figura en primer lugar, 2 para la que figura en tercero, 3 para la de la quinta posición, hasta 7 para la omitida, de menor importancia para esta persona como demuestra el hecho de que no la ha mencionado. 


\section{Validez estadística del ADM}

Antes de analizar el significado de la figura 2, es necesario, para que la interpretación que se haga tenga sentido, justificar la validez estadística del análisis, lo que haremos explicando tanto la forma en que se ha obtenido como las principales medidas estadísticas asociadas al mismo.

Aunque en un primer intento se trató de encontrar una solución no penalizada, los resultados mostraron que, si bien la solución obtenida era satisfactoria desde el punto de vista del ajuste a los datos iniciales existían indicios fuertes de degeneración, circunstancia bajo la cual no es posible ninguna interpretación de los mismos.

Para resolver este problema, según proponen Busing, Groenen y Heiser (2005), se optó por incluir la penalización en el análisis (con término de penalización de valor 0,3 y rango 1) tras probar con distintos valores para sus parámetros, eligiéndose los indicados por producir una solución aceptable tanto en términos de ajuste como de no degeneración, como puede comprobarse en la tabla 6, que presenta las principales medidas asociadas al análisis:

Tabla 6

Medidas asociadas al análisis PREFSCAL

\begin{tabular}{|c|c|c|c|c|}
\hline \multirow[b]{2}{*}{ Stress normalizado } & \multicolumn{2}{|c|}{ Coeficiente de variación } & \multirow{2}{*}{$\begin{array}{l}\text { Índice de } \\
\text { DeSarbo }\end{array}$} & \multirow{2}{*}{$\begin{array}{l}\text { Índice de } \\
\text { Shepard }\end{array}$} \\
\hline & $\begin{array}{c}\text { Proximidades } \\
\text { originales }\end{array}$ & $\begin{array}{l}\text { Proximidades } \\
\text { transformadas }\end{array}$ & & \\
\hline 0,073 & 0,536 & 0,681 & 0,066 & 0,794 \\
\hline
\end{tabular}

El coeficiente de Stress, próximo a cero, indica un buen ajuste, mientras que las cuatro medidas restantes señalan claros indicios de no degeneración en la solución: coeficientes de variación de similar magnitud para las proximidades originales y transformadas, un valor cercano a cero para el índice de Desarbo e índice de Shepard de prácticamente el 80\%. En definitiva, la representación obtenida es coherente con los datos originales, por lo que su interpretación puede serles trasladada.

Sobre los atributos, estos aparecen, como parece razonable, desagrupados. En efecto, si dos de ellos aparecieran muy próximos, debería interpretarse su cercanía como un signo de que ambos se refieren a un mismo rasgo que podría no ser siquiera ninguno de ellos.

Dentro de este aislamiento, merece la pena señalar algunos hechos que consideramos interesantes:

- La proximidad entre empleo, idioma y formación casi se comenta por sí misma: son los resultados tangibles deseados (formación e idioma) y su esperada consecuencia: trabajo. Geométricamente, parece intuirse que los tres aspectos están vinculados por importancia. 
- Esfuerzo e intercambio también son dos atributos que están muy alejados en la escala, aunque se establecen como referentes del público objeto de estudio, con clara influencia entre ambas del elemento cara.

- En estos dos atributos citados anteriormente, cabe destacar que el alumnado de centros públicos asocia el esfuerzo a la universidad privada; mientras que el otro grupo estudiado, el alumnado de centros privados vincula los intercambios.

- El atributo cara, otorgado a la universidad privada, parece ser unánime en su identidad, ya que se encuentra prácticamente en el centro del gráfico.

\section{Discusión y conclusiones}

La primera aportación (metodológica) derivada de este estudio estriba en denotar las posibilidades que aportan las RSN en investigaciones exploratorias en ciencias sociales que requieran captar la opinión de los sujetos, pues eliminan los apriorismos y permiten de forma sencilla sistematizar cómo los sujetos forman sus representaciones sobre ideas, comportamientos, productos, etc., si bien al precio de la ambigüedad de las respuestas, pues distintos sujetos pueden emplear un mismo término atribuyéndole significados o matices diferentes, lo que constituye una limitación del procedimiento que se traslada inevitablemente a nuestro trabajo.

La aplicación de las RSN es complementada con la técnica analítica multivariada despliegue multidimensional de lo que resulta un mecanismo adecuado para la triangulación con finalidad de validación.

En este contexto, los resultados son convergentes y nos llevan a extraer las siguientes conclusiones. Los estudiantes preuniversitarios definen la universidad privada como un servicio caro, con fuerte presencia de la formación y el esfuerzo, y notoriedad de los intercambios, empleo, prestigio e idioma. Por este orden y estos atributos son los que confirman la Hipótesis 1 planteada en este estudio. Porque independientemente de las características sociodemográficas de los encuestados, la mayoría de los individuos generan su imagen en torno a los mismos atributos.

Sin embargo, la segunda hipótesis se acepta en cierta medida. Por un lado, no se admite la Hipótesis 2a, ya que en principio se planteó que el sexo femenino tendría más riqueza semántica que el masculino, pero una vez presentada la RSN, el resultado es el contrario. La RSN compuesta por varones tiene una riqueza léxica superior a la representada por las mujeres; en concreto los hombres aportan siete atributos a su red, frente a los seis que aportan las mujeres. Se exponía la aceptación en cierta medida, porque, aunque se observa que la Hipótesis 2a no se acepta, no sucede lo mismo con la Hipótesis $2 b$, que sí se acepta al confirmar que los alumnos de procedencia privada o concertada aportan más valores positivos en la definición de la universidad privada. Específicamente, además de aportar más ítems, los aportados son más positivos, incorporando de forma concreta a esta afirmación los atributos empleo y futuro.

Estas consideraciones ofrecen algunas claves a las universidades privadas en materia de gestión y diferenciación. Ante el atributo cara, el más repetido y el que más relevancia tiene en los análisis propuestos, la universidad tiene que responder a través de valores que contrarresten y hagan menguar esta percepción; bien afrontándolo 
directamente, bien potenciando los atributos positivos que la población investigada valora alta y positivamente. Así, la implantación de sistemas de ayudas económicas destinadas a estudiantes con buenos expedientes y escasos recursos económicos sería un ejemplo de lo primero, mientras que la potenciación de la formación (por ejemplo, incrementando la atención al alumnado o mejorando la cualificación del profesorado), la internacionalización a través de convenios con otros centros en el extranjero y la mejora de la empleabilidad gracias a convenios con empresas son algunos ejemplos de lo segundo. El esfuerzo y el coste se verían claramente menguados si se consiguen potenciar los citados anteriormente, y que se consideran fortalezas.

En cuanto a la preferencia de atributos de los grupos estudiados, los chicos de centros privados se decantan por los intercambios, la formación y el idioma; mientras que los de centros públicos coinciden con los anteriormente citados solo en la formación, ya que para ellos además de este, el atributo de mayor peso es el esfuerzo. Las chicas de centros públicos por su parte otorgan la mayor relevancia, al igual que los varones de esta titularidad de centros, al esfuerzo. Las pertenecientes a centros privados por su parte, tienen más afijación sobre el empleo y el sistema de intercambio que diferencian a la universidad privada.

En definitiva, este trabajo aplica un procedimiento mixto novedoso (con elementos cualitativos y cuantitativos) que puede ser útil para el análisis de situaciones del ámbito educativo relacionadas con valoraciones o percepciones, permitiendo al investigador la cuantificación y visualización de los resultados derivados de las opiniones espontáneas de los participantes.

\section{Bibliografía}

Arpan, L. M., Raney, A. A. y Zivnuska, S. (2003). A cognitive approach to understanding university image. Corporate Communications: An International Journal, 8(2), 97-113.

Azorín, J. F. M., Gamero, M. D. L., Moliner, J. P., Ortega, E. M. P. y Guilló, J. J. T. (2012). Métodos híbridos de investigación y dirección de empresas: ventajas e implicaciones. Cuadernos de Economía y Dirección de la Empresa, 15(2), 55-62.

Barich, H. y Kotler, P. (1991). A framework for marketing image management. Sloan Management Review, 32(2), 94-104.

Beerli Palacio, A., Díaz Meneses, G. y Pérez Pérez, P. J. (2002). The configuration of the university image and its relationship with the satisfaction of students. Journal of Educational Administration, 40(5), 486-505.

Belanger, C., Mount, J. y Wilson, M. (2002). Institutional image and retention. Tertiary Education and Management, 8(3), 217-230.

Brown, R. M. y Mazzarol, T. W. (2009). The importance of institutional image to student satisfaction and loyalty within higher education. Higher Education, 58(1), 81-95.

Busing, F. M., Groenen, P. J. y Heiser, W. J. (2005). Avoiding degeneracy in multidimensional unfolding by penalizing on the coefficient of variation. Psychometrika, 70(1), 71-98.

Capriotti, P. (1999). Planificación estratégica de la imagen corporativa. Barcelona: Ariel. Recuperado de http://www.bidireccional.net/Blog/PEIC_4ed.pdf. 
Cernicova, M., Dragomir, M. y Palea, A. (2015). A Students' and Professors' View on the Image of Their University: case study: Politehnica University of Timisoara. ProcediaSocial and Behavioral Sciences, 191, 98-102. Recuperado de http://www.sciencedirect. com/science/article/pii/S187704281502755X.

Duarte, P. O., Alves, H. B. y Raposo, M. B. (2010). Understanding university image: a structural equation model approach. International Review on Public and Nonprofit Marketing, 7(1), 21-36.

Figueroa, J. G., González, E. G. y Solís, V. M. (1976). An approach to the problem of meaning: Semantic networks. Journal of Psycholinguistic Research, 5(2), 107-115.

Gómez, F. H. y Medina, R. Z. (2013). La triangulación interdisciplinar-metodológica (TIM) como modelo para analizar la imagen de las instituciones universitarias. En Investigar la Comunicación hoy. Revisión de politicas científicas y aportaciones metodológicas: Simposio Internacional sobre Política Científica en Comunicación (pp. 385-410). Facultad de Ciencias Sociales, Jurídicas y de la Comunicación. Recuperado de http:// uvadoc.uva.es/handle/10324/3042.

Gray, B. J., Shyan Fam, K. y Llanes, V. A. (2003). Branding universities in Asian markets. Journal of Product \& Brand Management, 12(2), 108-120.

Hair, J., Anderson, R., Tatham, R. y Black, W. (1999). Análisis multivariado. Madrid: Prentice Hall.

Harrison-Walker, L. J. (2011). Strategic positioning of nations as brands. Journal of International Business Research, 10(2), 135.

Hosseini, M. H. y Nahad, R. F. (2012). Investigating antecedents and consequences of open University brand image. International Journal of Academic Research, 4(4), 68-77.

Iqbal, M. J., Rasli, A. B. M. y Hassan, I. (2012). University branding: A myth or a reality. Pakistan Journal of Commerce and Social Sciences, 6(1), 168-184. Recuperado de https:// www.academia.edu/3713717/University_Branding_A_Myth_or_a_Reality.

Instituto Nacional de Estadística (España). INEbase [en línea]. [Madrid]: INE. Recuperado en http://www.ine.es/jaxi/Tabla.htm?path=/t13/p405/a2000-2001/10/\&file=02001. $\mathrm{p} x \& \mathrm{~L}=0$

Ivy, J. (2001). Higher education institution image: a correspondence analysis approach. International Journal of Educational Management, 15(6), 276-282.

Kazoleas, D., Kim, Y. y Anne Moffitt, M. (2001). Institutional image: a case study. Corporate Communications: An International journal, 6(4), 205-216.

Kotler, P., y Andreasen, A. (1996). Strategic Marketing for Nonprofit Organizations. Upper Saddle River, NJ: Prentice-Hall.

Luque-Martínez, T. y Del Barrio-García, S. (2007). Análisis del valor de las percepciones de los clientes en el diagnóstico estratégico de la universidad. Ponencia presentada en el International Congress Marketing Trends, Paris (pp. 26-27). Recuperado de http:// www.marketing-trends-congress.com/archives/2007/HTML/pages/strategie.htm.

Luque-Martínez, T., y Del Barrio-García, S. (2009). Modelling university image: The teaching staff viewpoint. Public Relations Review, 35(3), 325-327.

Mainardes, E. W., Alves, H. M. B. y Raposo, M. L. B. (2013). Identifying stakeholders in a Portuguese university: A case study. Revista de Educación, 362, 429-457. 
Mackelo, O. y Drūteikienè, G. (2010). The image of a higher education institution, its structure and hierarchical level: the case of the Vilnius University Faculty of Economics. Ekonomika, 89, 105-121.

Ministerio de Educación, Cultura y Deporte (MECD). (2015). Datos básicos del Sistema Universitario Español 2013-2014. Recuperado de http://www.mecd.gob.es/dms/mecd/ educacion-mecd/areas-educacion/universidades/estadisticas-informes/datos-cifras/ Datos-y-Cifras-del-SUE-Curso-2014-2015.pdf

Ortigueira Sánchez, M. y Ortigueira Bouzada, M. (2000). Las clases o servicios docentes universitarios: reflexiones en torno a su calidad y la calidad de su imagen. II Jornadas Andaluzas de Calidad en la Enseñanza Universitaria, Sevilla, (pp. 143-158). Instituto de Ciencias de la Educación.

Pampaloni, A. M. (2010). The influence of organizational image on college selection: what students seek in institutions of higher education. Journal of Marketing for Higher Education, 20(1), 19-48.

Reyes-Lagunes, I. (1993). Las Redes Semánticas Naturales, su conceptualización y su utilización en la construcción de instrumentos. Revista de Psicología Social y Personalidad, 9(1), 81-97.

Sabiote, C. R., Pérez, J. G. y Cano, A. F. (2004). Posibilidades del escalamiento multidimensional en la modelización de desajustes asociados a la reforma de planes de estudio universitarios. Revista de Investigación Educativa, 22(2), 377-391.

Sung, M. y Yang, S. U. (2008). Toward the model of university image: The influence of brand personality, external prestige, and reputation. Journal of Public Relations Research, 20(4), 357-376.

Valdez Medina, J. L., Gonzáles, E. S., Outhof, B. H. y Gonzáles Arrieta, L. F. N. I. (1998). Redes semánticas de valores y el sentido de la vida. La Psicología Social en México, 7, 456-460.

Vera Noriega, J. A., Eduardo Pimentel, C. y Batista de Albuquerque, F. J. (2005). Redes semánticas: aspectos teóricos, técnicos, metodológicos y analíticos. Ra Ximhai, 1(003). Recuperado de http://www.redalyc.org/articulo.oa?id=46110301.

Zermeño, A. I., Arellano, A. C. y Ramírez, V. A. (2005). Redes Semánticas Naturales: técnica para representar los significados que los jóvenes tienen sobre televisión, Internet y expectativas de vida. Estudios sobre las culturas contemporáneas, 11(22), 305-334.

Fecha de recepción: 29 de abril de 2016

Fecha de revisión: 29 de abril de 2016

Fecha de aceptación: 30 de mayo de 2017 
